\section{Técnica de overbooking no atendimento público ambulatorial em uma unidade do Sistema Único de Saúde}

\author{
Overbooking in an outpatient healthcare facility in \\ the Brazilian Unified National Health System
}

\author{
Práctica de overbooking en la atención \\ ambulatoria pública dentro de una unidad del \\ Sistema Único de Salud
}

\begin{abstract}
Management issues like resource optimization, cost cuts, quality gains, and improvements in care have become increasingly important and are essential to the sustainability of healthcare organizations. This study analyzed the efficiency of public outpatient care, where the high rate of patient no-show leads to low use of resources, while paradoxically the demand for medical care clearly exceeds the supply. A quantitative and descriptive approach analyzed the use of "overbooking" to reduce the effects of patient noshow in a public outpatient clinic. Using statistical and mathematical methods and based on patient and appointment data collected from a survey, an interesting comparison was made between levels of overbooking and the respective resource utilization rates and service levels for each scenario. The findings point to important gains in efficiency, thus demonstrating the potential of increasing the number of services in the same asset base.
\end{abstract}

Absenteeism; Ambulatory Health Services; Health Resources

\author{
Marcelo Oleskovicz 1 \\ Fábio Lotti Oliva 1 \\ Celso Cláudio de Hildebrand e Grisi 1 \\ Afonso Carneiro Lima 1 \\ Isaías Custódio ${ }^{1}$
}

\section{Resumo}

Aspectos como otimização de recursos, redução de custos, ganhos de qualidade e melhoria no atendimento receberam importância e são fundamentais para a sustentabilidade das organizações. Este trabalho analisa a eficiência no atendimento público ambulatorial, onde a alta taxa de absenteísmo por parte dos usuários implica baixa utilização de recursos, enquanto que, paradoxalmente, a demanda por atendimento médico é nitidamente superior à oferta. A abordagem, quantitativa e de caráter descritivo, analisa a utilização da técnica de overbooking para reduzir os efeitos do absenteísmo em um ambulatório da rede pública. Mediante o uso de métodos estatísticos e matemáticos, com base em dados de pacientes e agendamentos coletados em pesquisa, desenhou-se um interessante comparativo entre níveis de overbooking e respectivas taxas de utilização de recursos e níveis de serviço para cada cenário analisado. Os resultados obtidos apontam ganhos expressivos de eficiência, demonstrando o potencial de aumento do número de atendimentos para uma mesma base de ativos.

Absenteísmo; Serviços Ambulatoriais; Recursos em Saúde 


\section{Introdução}

O absenteísmo dos usuários da rede pública brasileira de saúde tem se revelado um problema crônico. O número de consultas e exames agendados e não realizados, devido ao não comparecimento dos pacientes, atinge índices significativamente altos e que podem ser percebidos em todas as regiões do Brasil e em diversos tipos de atendimento e especialidades médicas. Embora mais comumente usado para designar a falta dos colaboradores ao trabalho em uma organização, no presente trabalho o termo absenteísmo foi considerado como o não comparecimento dos usuários às consultas e exames agendados no setor de saúde, uma vez que é o termo adotado pelo ambulatório que é objeto deste estudo.

O índice de não comparecimentos de usuários às consultas e exames agendados no Sistema Único de Saúde (SUS) é significativamente alto, de forma geral próximo ou superior a $25 \%$. O número de não comparecimento está relacionado a diversos fatores 1 e varia dependendo da localidade, da especialidade médica e da faixa etária do paciente, por exemplo, clínicas no Estado do Amazonas apresentaram 37\% de não comparecimento em 2012 2; na rede pública do Grande ABC (São Paulo) verificou-se o índice de não comparecimento de 25\% em 2012 3; o mesmo índice de $25 \%$ foi constatado em Uberlândia (Minas Gerais) 4 . As informações disponíveis indicam que esse é um problema crônico e que se estende por todo o país 2,3,4,5,6,7,8,9, sendo esses fatores de contribuição para a relevância deste estudo.

As deficiências na gestão de serviços públicos contribuem para o agravamento da situação, uma vez que não são percebidas ações efetivas no sentido de reduzir o absenteísmo ou mitigar os seus impactos negativos. Cria-se assim um paradoxo, em que há escassez de recursos públicos e, ao mesmo tempo, a sua subutilização. Nesse cenário, ganhos de eficiência na administração de serviços públicos tornam-se fator crítico para alcançar melhores níveis de atendimento à população. Segundo Paiva 10, a introdução de inovações nos processos internos à administração pública ainda é incipiente e há um vasto campo para se avançar.

Um grande desafio seria como utilizar a tecnologia e as práticas de gestão privada para simplificar os processos e agilizar as decisões, sendo fundamental a superação de barreiras culturais. É inimaginável fazer mais e melhor sem mudar as práticas e os processos da administração 10 .

A técnica de overbooking, inserida nos modelos de gerenciamento da receita, têm ampla utilização em setores como o de transporte e o de hotelaria, porém ainda não tem seu potencial explorado em diversos outros contextos. Essa técnica tem demonstrado resultados significativos 11, com benefícios tanto para prestadores como para clientes/usuários, na medida em que os impactos negativos do não comparecimento (no show) de clientes/usuários de serviços são compensados. Ela permite uma melhor ocupação dos recursos, ao mesmo tempo em que disponibiliza mais capacidade para atendimento 12,13,14 . Assim, cabe investigar o quanto a utilização do overbooking pode contribuir para a melhor gestão de serviços públicos de saúde 15,16,17,18,19.

Dessa forma, o objetivo geral deste trabalho consiste em analisar a técnica de overbooking como uma alternativa para mitigar os impactos negativos do absenteísmo na rede pública brasileira de saúde. Esse objetivo geral pode ser dividido em dois objetivos específicos: (1) estimar o potencial dos ganhos de eficiência no atendimento das consultas ambulatoriais com o uso dessa ferramenta; e (2) propor um padrão de abordagem para análise de overbooking na área de saúde pública.

Em relação à delimitação do estudo, vale ressaltar que a busca por soluções às causas do absenteísmo não faz parte do escopo, uma vez que o foco incide somente sobre os seus efeitos.

Embora tenha seus conceitos largamente utilizados em determinados setores, a abordagem proposta ainda é incipiente na área de saúde no Brasil, bem como na administração pública como um todo, o que confere originalidade a este trabalho. $\mathrm{O}$ fato de existirem técnicas e ferramentas disponíveis, bem como a relativamente baixa complexidade de análise, figuram como importantes viabilizadores da proposta.

\section{Overbooking}

Segundo Bobb \& Veral 20, pesquisas sobre overbooking são a base para os estudos sobre gerenciamento da receita, iniciados pelo trabalho pioneiro de Beckmann \& Bobkowski 12 em modelagem estatística. Existem dois tipos principais de modelos de overbooking utilizados na prática: o primeiro é um modelo econômico básico, que determina o limite do overbooking no ponto onde a receita marginal esperada se iguala ao custo marginal 14 , ou seja, quando a receita adicional advinda com a prática de overbooking se iguala aos custos adicionais para suportá-la. O segundo e mais popular método tem como base um modelo de nível de serviço desejado similar ao utilizado na determinação de estoques de segurança para gerenciamento de inventário. Os conceitos centrais desse modelo são: nível de serviço ${ }^{21}$, 
o percentual de atendimento do serviço que se quer ofertar, estoque de segurança 13 , e estoque adicional para atender a uma demanda superior à previsão original de determinado período. $\mathrm{Na}$ presente pesquisa, utilizou-se o modelo de nível de serviço desejado.

Conforme Chopra \& Mendel 13, a tática de overbooking de um ativo disponível é aplicável em qualquer situação em que os usuários possam cancelar pedidos e o valor do ativo cai significativamente após um prazo, devido à sua perecibilidade. Se os cancelamentos podem ser previstos com precisão, o nível de overbooking pode ser facilmente determinado. Na prática, no entanto, os cancelamentos são incertos. O uso eficiente e eficaz dos recursos públicos tem sido item constante da pauta de interesse de uma sociedade democrática, assim sendo, a busca por uma utilização maximizada vai ao encontro dos anseios da coletividade 22,23,24.

O dilema básico a ser considerado durante o overbooking é entre ter capacidade (ou estoque) desperdiçada por excesso de cancelamentos ou ter falta de capacidade (ou estoque) devido a poucos cancelamentos, situação em que seria necessário um recurso extra, normalmente de alto custo. O custo da capacidade desperdiçada é o custo unitário da capacidade que não foi utilizada. O custo da falta de capacidade é o custo unitário resultante do uso de um recurso extra. $\mathrm{O}$ objetivo, ao decidir pelo overbooking, é maximizar o atendimento mediante a minimização do custo de capacidade desperdiçada e do custo de falta de capacidade 13 . Essa decisão pode ser desenvolvida em termos de uma fórmula que pode ser utilizada para definir os níveis de overbooking para um ativo. Define-se o custo marginal de se ter capacidade ociosa como $\mathrm{C}_{\mathrm{w}}$, e o custo marginal de se ter falta de capacidade como $\mathrm{C}_{\mathrm{s}}$. Denominemos $\mathrm{O}^{*}$ o nível ótimo de overbooking e $\mathrm{s}^{*}$ a probabilidade de os cancelamentos serem menores ou iguais a $\mathrm{O}^{*}$.

Dada a incerteza dos cancelamentos, existe uma distribuição para sua probabilidade. Se s* é a probabilidade de os cancelamentos serem menores ou iguais $\mathrm{O}^{*}$, então 1 -s* é a probabilidade de os cancelamentos serem maiores que O*. O nível ótimo de overbooking ocorre no ponto em que a redução no custo de oportunidade passa a ser igual ao custo do recurso extra, ou seja, $\mathrm{s}^{*}$.Cs $=\left(1-\mathrm{s}^{*}\right)$.Cw. De tal sorte que $\mathrm{s}^{*}=$ Prob $\left(\right.$ cancelamentos $\left.\leq \mathrm{O}^{*}\right)=\mathrm{Cw} /(\mathrm{Cw}+\mathrm{Cs})$. Considerando a distribuição dos cancelamentos como uma função do nível de reservas (capacidade $L+$ overbooking $O)$, tem-se $\mu(L+O)$ como média e $\sigma(L+O)$ como desvio padrão, sendo assim, o nível ótimo de overbooking dado s* é obtido pela equação $\mathrm{O}^{*} / \mathrm{s}^{*} \sim \mathrm{N}(\mu(\mathrm{L}+\mathrm{O}), \sigma(\mathrm{L}+\mathrm{O}))$.
Observe que o nível ótimo de overbooking deverá aumentar à medida que aumentar o custo por unidade não utilizada, e o nível de overbooking deverá diminuir à medida que aumentar o custo do recurso extra 16. Observe também que o nível de overbooking aumenta a utilização do ativo pelos usuários. O uso dessa prática diminui o número de usuários que são recusados e, portanto, melhora a disponibilidade de ativo para a sociedade ${ }^{13}$.

Conforme Chopra \& Mendel 13, estoque de segurança é o estoque mantido para satisfazer a demanda que excede a previsão para um determinado período. O estoque de segurança é mantido porque a demanda é incerta e a falta de um produto ou de um serviço pode ocorrer caso a demanda real ultrapasse a prevista. O nível adequado de estoque de segurança é determinado pelos seguintes fatores: incerteza tanto da demanda quanto da oferta, e o nível desejado de disponibilidade do produto ou serviço.

Segundo Ballou 21, o objetivo principal do gerenciamento de estoque é assegurar que o produto ou o serviço esteja disponível no tempo e nas quantidades desejadas. Geralmente é avaliado com base na probabilidade de ser capaz de preencher um pedido para um produto do estoque atual. Essa probabilidade, ou a taxa de preenchimento do item, é chamada de nível de serviço e, para apenas um item, pode ser definida como:

$$
\begin{gathered}
\text { Nível de serviço }=1 \text { - Número esperado de } \\
\text { unidades faltantes }
\end{gathered}
$$

Para a determinação do estoque de segurança em função do nível de serviço, utilizam-se as seguintes equações:

$\mathrm{NS}=1-\mathrm{ESC} / \mathrm{Q}$

em que, NS: Nível de Serviço, ESC: Número Esperado de Unidades Faltantes e Q: Quantidade do Lote (Demanda).

$\mathrm{ESC}=-\mathrm{ss}\left[1-\mathrm{F}_{\mathrm{s}}(\mathrm{ss} / \mathrm{dp})\right]+\sigma_{\mathrm{L}} \mathrm{f}_{\mathrm{x}}(\mathrm{ss} / \mathrm{dp})$

em que, ss: estoque de segurança, e $\sigma_{L}$ : desvio padrão da demanda.

Com o objetivo de viabilizar um modelo que seja independente das quantidades absolutas de vagas disponíveis e agendamentos - uma vez que estas mudam conforme especialidade, período e outras variáveis - os cálculos têm como referência os percentuais dos agendamentos (número de consultas agendadas).

Como utilizaremos o modelo baseado em Nível de Serviço e controle de estoque para as simulações de cenários, fez-se uma analogia entre as variáveis utilizadas em controle de estoque e as do caso estudado, representada na Tabela 1. 
Tabela 1

Analogia entre teoria de nível de serviço e caso.

\begin{tabular}{lccc}
\hline Símbolo & Controle de estoque & Unidade & Caso estudado \\
\hline Q & Demanda & Unidade & Taxa de comparecimento \\
esperada & Enidade & Erro padrão da taxa de \\
$\sigma$ & Desvio padrão da demanda & Unidade & comparecimento esperada \\
ESC & Unidades faltantes & Unidade & Número esperado de vagas \\
SS & Estoque de segurança & Unidade & Vagas de contingência \\
NS & Taxa de preenchimento & Unidade & Nível de serviço
\end{tabular}

\section{Metodologia}

Quanto à natureza da pesquisa, este trabalho consiste em uma pesquisa aplicada, em que o objetivo e o conhecimento adquirido são voltados à solução de um problema. A abordagem é quantitativa, pressupondo a quantificação do caso e solução, a fim de classificar e analisar de forma estatística o conjunto de dados obtidos 25 .

O estudo contemplou o Ambulatório de Especialidades Jardim Peri Peri (AE Jardim Peri Peri), Zona Oeste de São Paulo, gerido pela Fundação Faculdade de Medicina (FFM) da Universidade de São Paulo (USP), de acordo com um contrato de gestão realizado com a Prefeitura Municipal de São Paulo. Essa unidade realiza cerca de 4 mil atendimentos mensais, contando com 28 profissionais em 17 especialidades médicas, números considerados significativos e adequados para os objetivos do estudo. Pode-se considerar, ainda, que a unidade representa um ambulatório típico do SUS quanto à taxa de absenteísmo. O fato de atender a uma base populacional de 420 mil usuários também contribuiu para a escolha desse ambulatório.

Utilizou-se o modelo de overbooking com base em nível de serviço e numa abordagem de simulação, com testes de diferentes níveis de serviço. Segundo Ballou 21, utiliza-se um nível de serviço como restrição quando não há possibilidade de calcular valores de perda tanto para o excesso quanto a falta de um produto ou serviço. Para a geração de parâmetros para simulações, utilizaram-se os conceitos e fórmulas de nível de serviço e estoque de segurança.

\section{Coleta de dados}

Os dados coletados para a pesquisa, como informações cadastrais dos pacientes, especialida- des médicas e agendamentos, estão hospedados no Sistema Integrado de Gestão da Assistência à Saúde (SIGA). O banco de dados que abriga os dados do SIGA é administrado pela Secretaria Municipal da Saúde de São Paulo, e foram obtidos por consulta e relatórios no sistema, acessado por navegador de Internet, uma vez que a importação direta não foi autorizada. Essa forma de acesso é a mesma utilizada pelos funcionários do AE Jardim Peri Peri e das unidades básicas de saúde (UBS), que executam diariamente as atividades de cadastramento, agendamento e manutenção dos serviços.

A escolha da AE Jardim Peri Peri pode ser considerada como um caso emblemático, visto que esta unidade é gerida pela FFM. No mesmo local também se encontra uma unidade de urgência e emergência de baixas e médias complexidades (AMA Jardim Peri Peri). A originalidade para a escolha dessas unidades seriam os diferenciais apresentados, como o "Agente de Acolhimento" e a "Classificação de Risco". Vale ressaltar que durante o período de coleta de dados para o estudo, a gestão pela FFM ainda era incipiente e as práticas de agendamento, portanto, seguiam os padrões usuais.

O Agente de Acolhimento realiza esclarecimentos e orientações a respeito dos serviços, objetivando otimizar o tempo de permanência na unidade. A Classificação de Risco busca priorizar o atendimento em relação à gravidade ou sofrimento. Portanto, neste estudo visamos responder às características buscadas nessas unidades específicas; em trabalhos futuros será necessária a comparação entre outras unidades.

Foram selecionadas para o estudo as especialidades com grande volume de consultas agendadas para o período.

Para a seleção da amostra seguiu-se os critérios apresentados em Larson \& Farber 26 e Malho- 
tra 27. Segundo os autores, a população é o total de todas as contagens de interesse na pesquisa e a amostra um subconjunto da população. Assim, a população pesquisada foi composta pelos habitantes que residem próximos à unidade, isto é, na subprefeitura do Butantã com população aproximada de 420 mil pessoas.

O tamanho da amostra foi definido segundo o cálculo estatístico para amostras finitas 27 , considerando uma margem de erro de $5 \%, p$ e $q$ de $50 \%$ e um nível de $95 \%$ de confiança. Como resultado, obteve-se uma amostra total aproximada de 500 indivíduos a serem pesquisados. Diante dos riscos de não respostas a algumas perguntas e considerando a análise dos dados por especialidades, obteve-se uma amostra superior, de aproximadamente 1.500 pessoas, como o conjunto de usuários a serem entrevistados.

A coleta de dados referentes à ausência deuse no período de 10 dias úteis entre 26 de setembro de 2011 e 7 de outubro de 2011. Foram levantados os dados de 1.512 agendamentos de consulta, para 13 especialidades médicas. A verificação de presença/ausência foi realizada no próprio Ambulatório de Especialidades, devido à falta deste parâmetro no SIGA. A identificação de sazonalidade do absenteísmo não fez parte do escopo do estudo, portanto, a escolha do período atendeu aos objetivos da pesquisa, ou seja, a busca de um período de volume de pessoas constante e contínuo.

\section{Análise de dados}

A análise estatística foi centrada na taxa de comparecimento, definida como a proporção de consultas às quais os usuários comparecem em relação às consultas agendadas, e a taxa de absenteísmo, por consequência, é a proporção de ausências em consultas em relação aos agendamentos.

Para a análise dos dados foram selecionados parâmetros para pesquisa com usuários e busca no SIGA, tendo como principais critérios o tempo e a dificuldade para obtenção. Desse modo, obteve-se a relação de parâmetros a serem coletados para análise. Esses parâmetros e suas fontes estão representados na Tabela 2.

Na análise bivariada comparou-se a presença ou ausência à consulta com cada característica listada na Tabela 2, por meio do teste de quiquadrado de Pearson. Embora a quantidade de registros coletados seja expressiva, ao considerarmos a segmentação por especialidade médica o número de amostras por segmento é baixo. Essa segmentação é necessária, pois a ausência à consulta em uma especialidade não é compensada com o comparecimento em outra, já que são serviços distintos. Somando-se a isso o baixo número de variáveis correlacionadas, a análise de regressão não se mostrou viável.

Considerando o volume de dados obtidos, objetivando apresentar os dados mais expressivos, segmentou-se a amostra por cinco especialidades médicas: Ortopedia e Traumatologia, Oftalmologia, Neurologia, Endocrinologia e Urologia. Além disso, para cada segmento, identificou-se o horário da consulta, agrupando-se os dados pelos períodos da manhã e da tarde.

Utilizando-se os conceitos e fórmulas do referencial teórico, foram testados valores para nível de serviço e seus respectivos estoques de segurança. Mediante analogia, esses estoques de segurança calculados foram as quantidades de consultas que serão consideradas como contingência (reserva de capacidade) para absorver as variações do absenteísmo. Com base nessas variações e no nível de serviço sugerido, pode-se estimar a probabilidade de falta de capacidade de atendimento. Desse modo, são gerados diferentes cenários para simulações.

As simulações utilizaram o método de overbooking baseado em modelo de nível de serviço. Para cada cenário foram simulados diferentes níveis de serviço, resultando nas respectivas taxas de utilização e níveis de overbooking correspondentes. Também foram incluídas simulações sem vagas de contingência, bem como sem overbooking. Para cada cenário encontramos, inicialmente, uma análise sem vagas de contingência, ou seja, sem reserva de capacidade. Para esse caso o agendamento definido pelo nível de overbooking levará a um comparecimento esperado igual à capacidade. Em outro extremo foi simulada a condição sem overbooking, representando a

\section{Tabela 2}

Parâmetros e fonte dos dados analisados.

\begin{tabular}{ll}
\hline Parâmetro & Fonte \\
\hline Presença/Ausência & Coleta (AE Jardim Peri Peri) \\
Dia da semana & SIGA \\
Especialidade médica & SIGA \\
Tipo de consulta & SIGA \\
Sexo do paciente & SIGA \\
Hora marcada para a consulta & SIGA \\
Município de residência & SIGA \\
Unidade de referência & SIGA
\end{tabular}

AE Jardim Peri Peri: Ambulatório de Especialidades Jardim Peri Peri; SIGA: Sistema Integrado de Gestão da Assistência à Saúde. 
situação atual do ambulatório. Nesse caso o nível de serviço é de $100 \%$, uma vez que os comparecimentos agendados nunca irão superar as quantidades agendadas. A taxa esperada de utilização será a taxa esperada de comparecimento, uma vez que não há agendamentos acima da capacidade (overbooking).

Assim sendo, obteve-se um comparativo entre cenários que possibilita avaliar as vantagens e desvantagens de cada alternativa. Entre esses cenários será incluída a situação atual, ou seja, sem a aplicação de overbooking.

\section{Análise dos resultados}

As variáveis associadas significativamente com o comparecimento à consulta foram: especialidade médica ( $\mathrm{p}<0,0001)$, tipo da consulta ( $\mathrm{p}<$ $0,0001)$ e hora marcada para consulta $(\mathrm{p}<0,001)$. A Tabela 3 contém um resumo dos resultados da análise.

Entende-se que, mesmo tomando-se ações efetivas que reduzam significativamente o absenteísmo, este dificilmente será eliminado ou diminuído uma vez que parte das causas pode estar relacionada a fatores de difícil identificação, monitoramento e controle 28,29,30. Assim sendo, medidas no sentido de minimizar os efeitos do absenteísmo, foco deste trabalho, são complementares e tão importantes quanto as iniciativas no sentido de reduzi-lo.

\section{Resultados das simulações}

Quanto maior o absenteísmo , maior o potencial de ganhos em termos de taxa de utilização, sempre, entretanto, com uma redução no nível de serviço. Isso pode ser percebido na diferença das taxas de utilização esperada entre os extremos dos cenários, representadas na Tabela 4 .

Constata-se que os ganhos são mais significativos para situações de alto absenteísmo (baixa taxa de comparecimento esperada) e baixa variação (baixo erro padrão da taxa de comparecimento esperada). Uma análise adicional pode ser feita explorando-se os diferentes valores de erro padrão para as taxas de comparecimento esperadas de uma mesma especialidade em diferentes períodos (manhã/tarde), apontados na Tabela 3.

Assumindo-se a hipótese de que as agendas podem ser remanejadas de modo a concentrar especialidades em um determinado período, pode-se alterar os agendamentos de modo a buscar um desvio menor das taxas de comparecimento esperadas. Para que isso seja viável, considerase também que os médicos não trabalham em período integral. Mantendo o objetivo de utilizar os atuais recursos ambulatoriais, à toda alteração de período para uma especialidade deverá ser considerada alteração de outra, em igual quantidade de agendamentos e em período oposto. Dessa maneira, uma simulação passa a ser interessante à medida que a soma das diferenças de erro padrão com a inversão das agendas, seja positiva. Para tanto, analisa-se a inversão entre as especialidades, tendo as taxas de utilização esperadas como um indicador.

Como exemplo, uma simulação poderia ser feita com uma inversão de períodos entre as especialidades Ortopedia/Traumatologia, com transferência de consultas da manhã para a tarde, e Oftalmologia, tendo agendamentos alterados em igual quantidade da tarde para a manhã. Com base nos cálculos para essas especialidades, mantendo-se os mesmos níveis de serviço a $99 \%$ e, consequentemente, os demais indicadores, obtemos um comparativo representado na Tabela 5. Verifica-se que Ortopedia/Traumatologia manhã de $93,99 \%$ para $98,1 \%$ no período da tarde; Oftalmologia manhã 94,75\% para $96,17 \%$ tarde.

A inversão simulada permitiu um incremento médio de 3,2\% na taxa média de utilização para as vagas remanejadas. Caso seja interessante, em vez de taxa de utilização esperada, o ganho pode ser convertido em aumento do nível de serviço. $\mathrm{O}$ mesmo raciocínio pode ser estendido para outro tipo de segmentação, com base em influência de outras variáveis nos indicadores e flexibilidade de alterações.

\section{Considerações finais}

Em linha com os objetivos deste estudo, o modelo proposto fornece um padrão de abordagem para análise da aplicação de overbooking no sistema público de saúde. Considerando a dificuldade de identificar, monitorar e controlar os fatores que levam ao não comparecimento dos usuários no sistema público de saúde 28,29,30, a adoção de uma técnica de maximizar o uso dos recursos públicos vai ao encontro dos anseios da sociedade 22,23,24.

Ainda dentro dos propósitos do trabalho, a aplicação do modelo nos dados amostrais referentes ao AE Jardim Peri Peri aponta para um expressivo potencial de ganhos no trade-off entre taxa de utilização e nível de serviço. Para todos os cenários analisados existem alternativas que proporcionam um equilíbrio entre esses dois parâmetros. Nitidamente as perspectivas de incremento em resultados estão relacionadas aos altos índices de absenteísmo percebidos no 
Tabela 3

Análise de intervalo de confiança.

\begin{tabular}{|c|c|c|c|c|c|}
\hline \multirow[t]{2}{*}{ Especialidade } & \multirow[t]{2}{*}{ Período } & \multirow{2}{*}{$\begin{array}{c}\text { Taxa de } \\
\text { comparecimento } \\
\text { esperada }\end{array}$} & \multicolumn{2}{|c|}{ Intervalo } & \multirow[t]{2}{*}{$\mathrm{n}$} \\
\hline & & & $\begin{array}{l}\text { Limite } \\
\text { inferior }\end{array}$ & $\begin{array}{l}\text { Limite } \\
\text { superior }\end{array}$ & \\
\hline \multirow[t]{2}{*}{ Ortopedia e Traumatologia } & Manhã & 0,7353 & 0,6277 & 0,8429 & 68 \\
\hline & Tarde & 0,8074 & 0,7601 & 0,8547 & 270 \\
\hline \multirow[t]{2}{*}{ Oftalmologia } & Manhã & 0,6336 & 0,55 & 0,7172 & 131 \\
\hline & Tarde & 0,7642 & 0,682 & 0,8463 & 106 \\
\hline \multirow[t]{2}{*}{ Neurologia } & Manhã & 0,7609 & 0,672 & 0,8497 & 92 \\
\hline & Tarde & 0,8023 & 0,7164 & 0,8882 & 86 \\
\hline \multirow[t]{2}{*}{ Endocrinologia } & Manhã & $\begin{array}{l}\text { Amostragem } \\
\text { insuficiente }\end{array}$ & - & - & 10 \\
\hline & Tarde & 0,8131 & 0,738 & 0,8882 & 107 \\
\hline \multirow[t]{2}{*}{ Urologia } & Manhã & 0,6316 & 0,5024 & 0,7607 & 57 \\
\hline & Tarde & 0,8667 & 0,7781 & 0,9552 & 60 \\
\hline
\end{tabular}

Tabela 4

Taxa de comparecimento esperada versus variação da taxa de utilização esperada.

\begin{tabular}{|c|c|c|c|c|c|}
\hline Especialidade & Período & $\begin{array}{c}\text { Taxa de } \\
\text { comparecimento } \\
\text { esperado } \\
(\%)\end{array}$ & $\begin{array}{c}\text { Taxa de } \\
\text { utilização } \\
\text { esperada } \\
(\mathrm{NO}=0) \\
(\%)\end{array}$ & $\begin{array}{c}\text { Taxa de } \\
\text { utilização } \\
\text { esperada } \\
(\text { ss = 0) } \\
(\%)\end{array}$ & $\begin{array}{c}\text { Diferença } \\
\text { entre taxas } \\
\text { de utilização } \\
\text { esperada } \\
\text { (p.p.) }\end{array}$ \\
\hline Urologia & Manhã & 63,2 & 63,2 & 98,1 & 35,0 \\
\hline Oftalmologia & Manhã & 63,4 & 63,4 & 97,3 & 34,0 \\
\hline Ortopedia e Traumatologia & Manhã & 73,5 & 73,5 & 97,1 & 23,5 \\
\hline Neurologia & Manhã & 76,1 & 76,1 & 97,7 & 21,6 \\
\hline Oftalmologia & Tarde & 76,4 & 76,4 & 97,8 & 21,4 \\
\hline Neurologia & Tarde & 80,2 & 80,2 & 97,9 & 17,6 \\
\hline Ortopedia e Traumatologia & Tarde & 80,7 & 80,7 & 98,8 & 18,1 \\
\hline Endocrinologia & Tarde & 81,3 & 81,3 & 98,0 & 16,7 \\
\hline Urologia & Tarde & 86,7 & 86,7 & 95,9 & 9,3 \\
\hline
\end{tabular}

Tabela 5

Análise de inversão de períodos entre especialidades médicas.

Taxa esperada de utilização (\%)

Nível de serviço $=99 \%$

\begin{tabular}{lccc} 
& Manhã & Tarde & Diferença após inversão \\
\hline Ortopedia/Traumatologia & 93,99 & 98,61 & 4,62 \\
Oftalmologia & 94,75 & 96,17 & $-1,42$ \\
& & Ganho $==>>$ & 3,20 \\
\hline
\end{tabular}


ambulatório, bem como suas variações relativamente baixas.

Nesse sentido, os resultados do trabalho contribuem para o aprofundamento dos estudos e novas abordagens. Destaca-se o benchmark com os setores aéreo e hoteleiro, em que a técnica de overbooking é amplamente utilizada. Uma análise econômica, incluindo aspectos como custos e a inclusão de viabilizadores qualitativos também se enquadram em interessantes alternativas.

Algumas limitações deste estudo devem ser registradas para contribuir com futuros trabalhos em diferentes contextos. A falta de informações nos sistemas de controle fez com que fosse complementada a base de dados da pesquisa com a coleta de campo, no caso específico, o compare- cimento ou não dos pacientes às consultas. Da mesma forma, a adoção de amostra estratificada por especialidades médicas proporcionaria um estudo mais completo, no entanto, demandaria uma pesquisa de campo mais extensa que o ambiente de estudo por vezes limita.

Quanto aos aspectos estatísticos, ainda há espaço para aprimoramento, que somente seria possível com uma base de dados mais ampla, permitindo a exploração de métodos como os de relações causais e séries temporais 31,32 . Apesar desses métodos não implicarem alterações significativas ao modelo matemático, um modelo estatístico mais refinado traria maior precisão aos resultados. Desse modo, recomenda-se a evolução da abordagem quantitativa para futuros trabalhos.

\section{Resumen}

Aspectos de gestión, tales como la optimización de recursos, reducción de costes, mejora de la calidad y servicio han adquirido cada vez más importancia y son fundamentales para mantener organizaciones sosteniblemente. Esta investigación analiza la eficiencia del servicio médico ambulatorio público, donde no se presenta una alta tasa de pacientes, lo que lleva a una baja utilización de los recursos existentes, mientras que, paradójicamente, la demanda de servicios médicos es claramente superior a su disponibilidad. El enfoque cuantitativo y descriptivo analiza la utilización de un exceso de reservas técnicas, con el fin de reducir el efecto de pacientes que no se dirigen, finalmente, a un servicio médico público ambulatorio. Se aplicaron métodos estadísticos y matemáticos en la información proporcionada por pacientes y en la recogida de información de la base de datos de consultas, que dieron lugar a una interesante comparación entre los niveles de exceso de reservas y su tasa de utilización, respecto a los recursos y niveles de servicio dentro de cada uno de los escenarios analizados. Los resultados obtenidos muestran un aumento significativo en la eficiencia.

Absenteismo; Servicios Ambulatorios de Salud;

Recursos en Salud

\section{Colaboradores}

M. Oleskovicz contribuiu com a coleta de dados e redigiu o texto. F. L. Oliva contribuiu com a análise dos dados, redação e ajustes no texto. C. C. H. Grisi e I. Custódio contribuíram com a revisão do texto final. A. C. Lima contribuiu com a redação do texto.

\section{Agradecimentos}

À gestão do Ambulatório de Especialidades Jardim Peri Peri, Zona Oeste de São Paulo, gerido pela Fundação Faculdade de Medicina da Universidade de São Paulo, que facilitou o acesso aos dados utilizados na pesquisa. 


\section{Referências}

1. Shonick W, Klein BW. An approach to reducing the adverse effects of broken appointments in primary care systems. Med Care 1977; 15:419-29.

2. Portal do Governo do Estado do Amazonas. Índice de pacientes faltosos chegou a $37 \%$ na Policlínica Codajás e unidade alerta para os prejuízos ao próprio usuário. http://www.amazonas.am.gov.br/ (acessado em 21/Jan/2013).

3. Moraes T. Pacientes faltam a $25 \%$ das consultas do SUS. Diário do Grande ABC 2013; 24 mar.

4. Fernandes A. Uma a cada 4 pessoas não vai às consultas. Correio de Uberlândia 2011; 6 nov.

5. HNEWS. Em cada três consultas gratuitas saúde pública registra uma falta: Secretaria de Saúde divulgou ontem um levantamento apontando para o elevado índice de faltas de pacientes em consultas especializadas. http://www.hnews.com.br (acessado em 07/Nov/2011).

6. Jornal Voz Ativa. Pacientes deixam de realizar 1800 exames agendados todos os anos em Ouro PretoMG. http://jornalvozativa.com (acessado em 01/ Nov/2011).

7. Jornal VS (Novo Hamburgo). Tarcísio admite dificuldades na saúde e anuncia melhorias. http:// www.jornalvs.com.br (acessado em 07/Nov/2011).

8. Pereira W. População está em déficit com a Saúde. Jornal Tribuna do Interior. http://www.itribuna. com.br (acessado em 28/Out/2011).

9. Toledo K. Aparelhos de ultrassom em SP têm ociosidade de $40 \%$ : a espera é de 3 meses. O Estado de S. Paulo 2011; 9 ago.

10. Paiva P. Inovação em gestão pública. O Estado de S. Paulo 2011; 5 mai

11. Moussawi-Haidar L, Cakanyildirim M. Optimal overbooking limits of a two-dimensional cargo problem: a profit maximization approach. Journal of Revenue and Pricing Management 2012; 11:453-76

12. Beckmann J, Bobkowski F. Airline demand: an analysis of some frequency distributions. Wilmington: Wiley Periodicals; 1986.

13. Chopra S, Meindel P. Supply chain management: strategy, planning and operation. Boston: Prentice Hall; 2010.

14. Littlewood K. Forecasting and control of passanger bookings. Journal of Revenue and Pricing Management 2005; 4:111-23.

15. Bean AB, Talaga J. Predicting appointment breaking. J Health Care Mark 1995; 15:29-34.

16. Campbell JD, Chez RA, Queen T, Barcelo A, Patron E. The no-show rate in a high risk obstetric clinic. J Womens Health Gend Based Med 2000; 9:891-5.

17. Dove HG, Schneider KC. The usefulness of patients' individual characteristics in predicting no-shows in outpatient clinics. Med Care 1981; 19:734-74
18. Garuda SR, Javalgi RG, Talluri VS. Tackling no-show behavior: a market-driven approach. Health Mark Q 1988; 5:25-45.

19. Sharp DJ, Hamilton W. Non-attendance at general practices and outpatients clinics: local systems are needed to address local problems. BMJ 2001; 323:1081-2.

20. Bobb L, Veral E. Open issues and future direction in revenue management. Journal of Revenue and Pricing Management 2008; 7:291-301.

21. Ballou RH. Gerenciamento da cadeia de suprimentos. Planejamento, organização e logística empresarial. Porto Alegre: Bookman; 2003.

22. Vanni T, Luz PM, Ribeiro RA, Novaes HMD, Polanczyk CA. Avaliação econômica em saúde: aplicações em doenças infecciosas. Cad Saúde Pública 2009; 25:2543-52.

23. Camelo Junior JS, Fernandes MIM, Jorge SM, Maciel LMZ, Santos JLF, Camargo Jr. AS, et al. Avaliação econômica em saúde: triagem neonatal da galactosemia. Cad Saúde Pública 2011; 27:666-76.

24. Fonseca PC, Ferreira MAM. Investigação dos níveis de eficiência na utilização de recursos no setor de saúde: uma análise das microrregiões de Minas Gerais. Saúde Soc 2009; 18:199-213.

25. Gil AC. Como elaborar projetos de pesquisa. 4a Ed. São Paulo: Editora Atlas; 2002.

26. Larson R, Farber B. Estatística aplicada. 2a Ed. São Paulo: Prentice Hall; 2004.

27. Malhotra N. Pesquisa de marketing: uma orientação aplicada. Porto Alegre: Bookman; 2001.

28. George A, Rubin G. Non-attendance in general practice: a systematic review and its implications for access to primary health care. Fam Pract 2003; 20:178-84.

29. Martin C, Perfect T, Mantle G. Non-attendance in primary care: the views of patients and practices on its causes, impact and solutions. Fam Pract 2005; $22: 638-43$.

30. Cashman SB, Savageau JA, Lemay CA, Ferguson W. Patient health status and appointment keeping in an urban community health center. J Health Care Poor Underserved 2004; 15:474-88.

31. Muthuramana K, Lawleyb M. A stochastic overbooking model for outpatient clinical scheduling with no-shows. IIE Trans 2008; 40:820-37.

32. Lina J, Muthuramanb K, Lawleya M. Optimal and approximate algorithms for sequential clinical scheduling with no-shows. IIE Trans Healthc Syst Eng 2011; 1:20-36.

Recebido em 30/Out/2012

Versão final reapresentada em 10/Nov/2013

Aprovado em 19/Nov/2013 